\title{
Experiência na Avaliação de Efetividade de um Jogo para Ensino de Conceitos de Requisitos em Sistemas Ubíquos
}

\author{
Rodrigo Santos, Thaiana Lima, Beatriz Campos, Cláudia Werner \\ COPPE/UFRJ, Universidade Federal do Rio de Janeiro, Brasil \\ Caixa Postal 68511 - CEP 21945-970 - Rio de Janeiro, RJ \\ \{rps, thaiana, biapc, werner\}@cos.ufrj.br
}

\begin{abstract}
The use of games as a supporting tool in the teaching and learning process has become common in different areas of knowledge. In this sense, a concern is emerging in the development of games in Software Engineering (SE) area, considering steps, and artifacts and features identification, towards producing a didactic and interesting game. From previous researches on developing games to SE, we present an experience on evaluating a game for teaching concepts related to requirements in ubiquitous systems, called UbiRE. The focus is on planning and executing an effectiveness evaluation.
\end{abstract}

Resumo. O uso de jogos como ferramenta de auxílio no processo de ensino e aprendizagem tem se tornado comum em diferentes áreas do conhecimento. Neste sentido, surge uma preocupação no desenvolvimento de jogos na área de Engenharia de Software (ES), considerando suas etapas e a forma de identificar artefatos e características que tornem o jogo mais didático e interessante. Partindo de pesquisas prévias sobre processo de desenvolvimento de jogos para ES, apresentamos a experiência na avaliação do jogo para ensino de conceitos de requisitos em sistemas ubíquos, denominado UbiRE. O enfoque está sobre uma avaliação de efetividade.

\section{Introdução}

A utilização de jogos como ferramentas educacionais tem crescido [Akilli 2007]. Isso se deve à popularização dos jogos, acesso facilitado a Internet, além das características intrínsecas a um jogo, que podem ser exploradas a fim de expor um conteúdo didático, de forma mais agradável [Savi et al., 2011]. Entre essas características, podemos citar a interação do jogador, uso de metáforas e a capacidade do jogo de simular qualquer conceito em um mundo virtual. Ou seja, tem-se uma ferramenta poderosa para motivar o processo de aprendizagem dos alunos e agregar realismo à experiência dos jogadores [von Wangenheim et al., 2009]. Na área de Engenharia de Software (ES), a pesquisa em educação vem se destacando [Lethbridge et al., 2007], tendo em vista que ela envolve conceitos complexos e muitas atividades práticas, que podem ser simulados no mundo virtual [Werner et al., 2009]. Entretanto, a ES muitas vezes não é vista como um tema alvo para desenvolvimento de jogos, principalmente se comparada a outras áreas [Lima et al., 2012]. O objetivo deste artigo é discutir a experiência na avaliação do jogo para ensino de conceitos de requisitos em sistemas ubíquos denominado UbiRE. A contribuição destaca neste artigo está sobre o planejamento e execução de uma avaliação de efetividade de jogos educacionais. Na Seção 2, apresentamos o UbiRE; na Seção 3, discutimos a avaliação de efetividade; e na Seção 4, concluímos o artigo. 


\section{O Jogo UbiRE}

Desenvolvido em uma das frentes de pesquisa do Projeto Tec3ES (Tecnologias e Estratégias para Educação em Engenharia de Software [Werner et al., 2009], o jogo $\mathrm{UbiRE}^{1}$ foi construído a partir de pesquisas sobre processo de desenvolvimento de jogos educacionais [Lima et al., 2012], cujo tema aborda ubiquidade e engenharia de requisitos (ER). O jogo acontece em uma casa (ambiente), onde a meta do jogador é "desvendar" e montar o sistema inteligente e ubíquo a partir da conexão entre equipamentos, móveis e utensílios (ações), desde que atendam aos requisitos do morador [Campos et al., 2011] para passar de fase, representadas pelos cômodos da casa. Ao final de cada fase, o jogo segue para uma tela de simulação, onde é possível verificar a funcionalidade de cada conexão e permitir que o morador valide as vantagens trazidas pelo sistema em um cômodo. Esta verificação se dá por meio de sensores próximos dos objetos, que são ativados quando o personagem se aproxima (Figura 1). Maiores detalhes são apresentados em [Lima et al., 2012].

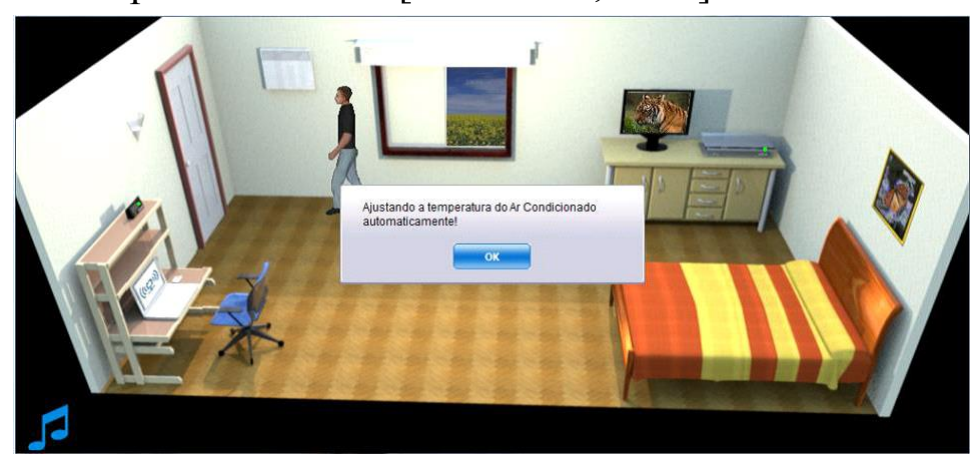

Figura 1. Simulação da Fase Quarto

\section{Avaliação de Efetividade}

Foi elaborado um estudo de avaliação de efetividade, que pudesse verificar o quanto a utilização do jogo auxiliaria no entendimento dos temas abordados. Testes sobre os temas envolvidos (requisitos e ubiquidade) foram aplicados, além de (i) breve treinamento sobre os mesmos temas, (ii) questionários de feedback e (iii) caracterização de cada participante. Esta avaliação visou responder a seguinte questão de pesquisa: " $O$ jogo UbiRE contribui para o aprendizado dos alunos?". Foi elaborado um plano contendo os detalhes da avaliação, incluindo todos os formulários e testes utilizados. Os alunos responderam os testes e questionários em formulários eletrônicos na Internet. As respostas foram coletadas e depositadas em uma planilha preparada com funções e fórmulas para calcular a pontuação de cada participante. $O$ estudo foi realizado com 9 alunos do $5^{\circ}$ módulo do Curso Superior de Tecnologia em Análise e Desenvolvimento de Sistemas oferecido pelo Instituto Federal de Educação, Ciência e Tecnologia do Piauí (IFPI) - Campus Teresina Central, com apoio remoto da Equipe de Reutilização de Software no Laboratório de Engenharia de Software (LENS) da COPPE/UFRJ.

\subsection{Metodologia Aplicada}

Os alunos participantes foram divididos homogeneamente em dois grupos, Grupo Experimental (Grupo A) e Grupo de Controle (Grupo B). Os grupos se diferiam pelo

\footnotetext{
${ }^{1}$ Disponível em $<$ http://www.cos.ufrj.br/ thaiana/ubire $>$.
} 
jogo utilizado: o Grupo Experimental jogou o UbiRE, e o Grupo de Controle, um outro jogo que não possui o mesmo tema, sendo considerado placebo. Ambos receberam um "Termo de Consentimento" e um "Formulário de Caracterização do Participante". Este segundo formulário foi preenchido com informações pessoais e acadêmicas, consideradas para posterior divisão dos grupos. Após o seu preenchimento, os participantes receberam um breve treinamento sobre os temas abordados no jogo UbiRE. Buscou-se assim reduzir a discrepância no nível de conhecimento entre eles.

Em seguida, os participantes realizaram um "Pré-teste" para medir o quanto o aluno aprendeu com o treinamento. Os resultados também contribuíram para a divisão dos alunos nos grupos, para que começassem a jogar o UbiRE (ou o placebo). Após isso, foi aplicado um "Pós-teste" com questões semelhantes às do primeiro teste, em termos de grau de dificuldade e conceitos envolvidos. No final, somente os alunos do Grupo Experimental receberam um questionário específico com perguntas sobre outros aspectos do UbiRE, incluindo observações, críticas e sugestões, derivado da proposta de [Savi et al., 2011]. Apesar de utilizarem diferentes jogos, os testes foram iguais para os grupos. Ao final da coleta, foi feito o levantamento das pontuações.

\subsection{Realização do Experimento}

Antes de iniciar qualquer procedimento do estudo, foram explicadas para os alunos as características da pesquisa. Em seguida, os participantes responderam às questões do formulário de caracterização. Somando a pontuação obtida em cada questão do formulário, o aluno recebeu uma nota de no máximo 5 pontos, representado no cálculo da pontuação total pela variável $C$ (Eq. 1). O treinamento se deu pela exposição de conteúdo teórico sobre os temas abordados no UbiRE por aproximadamente 20 minutos e foi realizada por um dos pesquisadores presentes do IFPI. A primeira avaliação possuía questões de múltipla escolha visando reduzir o tempo gasto e maximizar a objetividade. Posteriormente, a pontuação foi utilizada para a alocação dos grupos, visando equilibrar a composição de ambos. A distribuição e ponderação dos pontos foram feitas de acordo com a equação Eq. 1:

onde:

$$
R=0.5 * T 1+C
$$

T1: Nota do "Pré-teste" $(0 . .10)$

$C$ : Nota referente ao formulário de caracterização $(0 . .5)$

Com os dados exportados para a planilha eletrônica já preparada com funções construídas em implementação VBA (Visual Basic for Applications), a separação dos grupos foi realizada de maneira imediata. A partir do valor de $R$, os alunos foram listados em ordem crescente. Os alunos posicionados nas classificações ímpares foram para o Grupo Experimental e nas pares para o Grupo de Controle. Dessa forma, buscouse equilíbrio entre o nível de conhecimento do grupo. A segunda avaliação foi feita a partir de dez questões, cada uma valeu um ponto. Esta avaliação visou verificar o quanto o jogo ajudou ao aluno a entender os conceitos. Ao final, os resultados da avaliação foram utilizados para avaliar a hipótese derivada da questão de pesquisa: "O UbiRE colabora para o entendimento dos temas nele abordados". Para isso, os desempenhos dos Grupos Experimental e de Controle foram comparados. 


\subsection{Resultados}

Foram comparadas as notas de cada aluno, de ambos os grupos, nos dois testes aplicados, a fim de verificar se aqueles que jogaram o UbiRE tiveram um aumento significativo na nota em relação aos que utilizaram o placebo. A partir das informações de caracterização dos participantes, foram destacadas algumas observações iniciais. $100 \%$ dos alunos declararam gastar no mínimo 20 horas semanais em jogos e no computador (extraclasse). Isto pode ser visto como um ponto positivo porque, quanto maior o nível de conforto do aluno com a tecnologia, mais facilmente ela pode ser inserida em sua vida acadêmica [von Wangenheim et al., 2009]. 33\% dos participantes havia cursado o Ensino Médio comum, enquanto a maioria cursou o Ensino Médio com Técnico em Informática, tendo cursado a disciplina de ES.

A Figura 2 exibe o nível de conhecimento dos alunos em relação aos temas que formam a base do UbiRE: ES (geral), ER e ubiquidade. Pode-se observar que o tema da ubiquidade é o mais desconhecido pela maioria dos alunos. A ES é em geral a mais aplicada pelos participantes em projetos, tanto acadêmicos quanto profissionais. A ER é conhecida pela maioria, e é mais aplicada em projetos acadêmicos. Partindo destas observações, ressalta-se a contribuição da pesquisa em jogos para ER por utilizar um tema importante para a indústria da ES [Boehm 2006] e de domínio mais complicado, em se tratando da formação dos profissionais de desenvolvimento de software, normalmente mais técnica [Lethbridge et al., 2007].

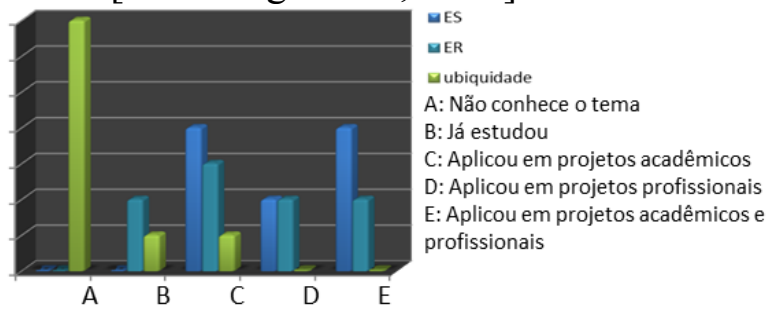

Figura 2. Distribuição dos participantes por conhecimento dos temas

A Figura 3 apresenta as notas dos dois testes: do lado esquerdo, o Grupo de Controle, e do lado direito, o Grupo Experimental. Pode-se observar que, no caso do Grupo de Controle, houve queda na nota de um participante, o que não aconteceu com nenhum participante do Grupo Experimental. A média do "Pré-teste" do Grupo de Controle foi 4,25, e a do Grupo Experimental foi 4,8. No Pós-teste, a média do Grupo de Controle foi 5,0, ao passo que a do Grupo Experimental foi 5,8. O aumento da média foi de $17,6 \%$ no primeiro caso e de $20,8 \%$ no segundo caso. O participante que obteve o maior aumento na nota depois do uso do jogo pertencia ao Grupo Experimental. Este aluno aumentou em $100 \%$ sua nota (de 3 para 6 pontos). Nenhum participante do Grupo de Controle obteve nota maior que os do Grupo Experimental no Pós-teste.
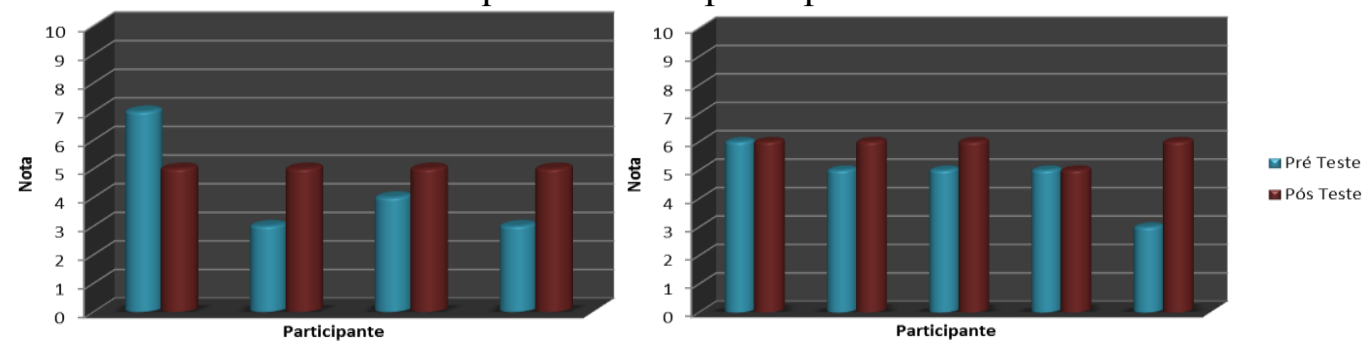

Figura 3. Resultados do Grupo Controle (esquerda) e do Experimental (direita) 
O questionário de feedback foi respondido pelos alunos do Grupo Experimental. Observou-se que as questões com menor média foram relativas a "Eu não percebi o tempo passar enquanto jogava, quando vi o jogo acabou" e "Senti-me mais no ambiente do jogo do que no mundo real, esquecendo-se do que estava ao meu redor". As respostas indicaram a necessidade de maior esforço em imergir o aluno no ambiente do jogo. Estas questões estão no documento "Plano de Estudos", extraídas de [Savi et al., 2011].

\section{Conclusão}

A utilização de jogos como estratégia para o processo de ensino e aprendizado vem se destacando. A pesquisa em Educação em ES tem se utilizado dos jogos, visto que eles podem representar um ambiente de simulação de conceitos de difícil reprodução em sala de aula. Este artigo apresentou um estudo de avaliação de efetividade realizado em sala de aula com o jogo UbiRE. O estudo foi planejado e documentado de acordo com experimentos relatados na literatura de Educação em ES. Foram obtidos resultados satisfatórios para o porte dos estudos e para o objetivo do jogo UbiRE. Como trabalhos futuros, pretende-se repetir o estudo em outras turmas e com um maior número de participantes. Além disso, pretende-se desenvolver uma metodologia para combinar a avaliação de usabilidade e de efetividade a fim de apoiar a ES aplicada à Informática na Educação, especialmente a avaliação de jogos educacionais.

Agradecimentos. Os autores agradecem ao CNPq, CAPES e FAPERJ pelo apoio financeiro. Os autores agradecem ainda à profa. Valéria Costa do IFPI pelo apoio na execução do estudo.

\section{Referências}

Akilli, G. (2007) "Games and Simulations: A new approach in education". In: Gibson, D., Aldrich, C., Prensky, M. (eds.) Games and simulations in online learning: research and development frameworks. Information Science Publishing, Hershey/PA, pp. 1-20.

Boehm, B. (2006) "A View of 20th and 21st Century Software Engineering". In: 28th International Conference on Software Engineering, Shanghai, China, pp. 12-29.

Campos, B., Lima, T., Santos, R., Werner, C., Limoeiro, F. (2011) "Experiência de Projeto e Desenvolvimento de Jogo para Ensino de Engenharia de Requisitos para Sistemas Ubíquos". In: XXII Simpósio Brasileiro de Informática na Educação, Aracaju, Brasil, pp. 960-963.

Lethbridge, T., Diaz-Herrera, J, LeBlanc, R, Thompson, J. (2007) "Improving Software Practice through Education: Challenges and Future Trends". In: 29th International Conference on Software Engineering, Minneapolis, USA, pp. 12-28.

Lima, T., Campos, B., Santos, R., Werner, C. (2012) "UbiRE: A Game for Teaching Requirements in the Context of Ubiquitous Systems". In: XXXVIII Latin American Informatics Conference (CLEI), XX CIESC, Medellín, Colombia, pp. 1-10.

Savi, R., Von Wangenheim, C., Borgatto, A. (2011) "Um Modelo de Avaliação de Jogos Educacionais na Engenharia de Software". In: XXV Simpósio Brasileiro de Engenharia de Software, São Paulo, Brasil, pp. 194-203.

von Wangenheim, C., Kochanski, D., Savi, R. (2009) "Revisão Sistemática sobre Avaliação de Jogos Voltados para Aprendizagem de Engenharia de Software no Brasil”. In: II Fórum de Educação em Engenharia de Software, XXIII SBES, Fortaleza, Brasil, pp. 41-48.

Werner, C., Rodrigues, C., Santos, R., Costa, H., Santo, R., Castro, W. (2009) "Projeto Tec3ES: Tecnologias e Estratégias para Educação em Engenharia de Software". In: XXXV Latin American Informatics Conference (CLEI), XXVII CIESC, Pelotas, Brasil, pp. 1-2. 